\title{
Dynamical evolution driven by bars and interactions : Input from numerical simulations
}

\author{
E. Athanassoula \\ Observatoire de Marseille, \\ 2 Place Le Verrier, \\ 13248 Marseille cedex 04, \\ France
}

\begin{abstract}
We discuss the evolution of a disc galaxy due to the formation of a bar and, subsequently, a peanut. After the formation stage there is still considerable evolution, albeit slower. In purely stellar cases the pattern speed of the bar decreases with time, while its amplitude grows. However, if a considerable gaseous component is present in the disc, the pattern speed may increase with time, while the bar strength may decrease. In some cases the gas can be brought sufficiently close to the center to create a strong central concentration, which, in turn, may modify the properties of the bar.

More violent evolution can take place during interactions, so that some disc substructures can be either formed or destroyed in a time scale which is small compared to a Hubble time. These include spirals, bars, bridges, tails, rings, thick discs and bulges. In some cases interactions may lead to mergings. We briefly review comparisons of the properties of merger remnants with those of elliptical galaxies, both for the case of pairwise mergings and the case of multiple mergings.
\end{abstract}

Keywords: disc galaxies, barred galaxies, bars, interactions, mergings, elliptical formation

\section{Introduction}

Many studies, and particularly those based on numerical simulations including time-dependence, have shown that disc galaxies can be considered as static, or even quasi-stationary, only in a zeroth order approximation and in fact undergo considerable evolution with time. We will here discuss first the evolution linked to a bar, namely its formation, the formation of a peanut and the subsequent slower secular evolution, which leads to changes in the bar pattern speed and strength (section 2 ). We will then turn to the more violent evolution which occurs during interactions and mergings (section 3). In particular we will discuss the formations of tails and bridges (section 3.1), the formation of collisional ring galaxies (section 3.2), the interaction of a barred galaxy with a small spheroidal companion (section 3.3), and the properties of merger remnants, including those of multiple mergers (section 3.4 ). 


\section{Bars as engines of secular evolution}

\subsection{BAR FORMATION}

The early seventies brought the realization that galactic discs are unstable to the formation of a bar, unless specific care is taken to stabilise them, or rather to reduce the growth rate of the instability (e.g. Athanassoula, 1984 or Sellwood \& Wilkinson, 1993 for reviews). As it grows, the bar brings considerable changes to the orbital structure of the disc. In an axisymmetric disc with not too high radial velocity dispersion, the orbits can be considered quasi-circular, with deviations that can approximated by epicycles (Binney \& Tremaine, 1987). After the growth of the bar most stars in the bar region are trapped around $x_{1}$ type orbits, i.e. around periodic orbits elongated along the bar (see e.g. Contopoulos \& Grosb $\varnothing 1,1989)$. Such orbits can not extend beyond corotation (hereafter CR), thus setting a natural limit to the length of the bar (Contopoulos, 1980). Furthermore, the outermost parts of the bar, just within corotation, are mainly populated by chaotic orbits and by orbits trapped around periodic orbits of type $n: 1$ (i.e. having $n$ radial oscillations for one rotation around the galactic center). Thus the $x_{1}$ orbits may fall short before CR. This led Skokos, Patsis \& Athanassoula (2002b) to argue that the bar should stop at the 4:1 resonance, or inner Ultra-harmonic resonance (hereafter iUHR). Athanassoula (1992) found from hydrodynamical simulation that the gas response agrees with observations only if the $\mathrm{CR}$ radius is $1.2 \pm$ 0.2 times the length of the bar semi-major axis, and this limit is in good agreement with whatever observational constraints are available (Athanassoula, 1992; Elmegreen, 1996). In fact the two criteria could well give the same limits for the bar length, although it is not easy to calculate the iUHR radius for an object as far from axisymmetry as a strongly barred disc galaxy.

After the bar has become sufficiently strong it will in turn be prey to another instability, the buckling instability, which will bend the orbits and bring them well outside the equatorial plane. As a result of this the bar will thicken and take a peanut shape. This is clearly seen in 3D simulations of bar unstable discs (e.g. Combes \& Sanders, 1981; Combes et al., 1990; Raha et al., 1991). Again there is a considerable change in the orbital structure after the peanut has grown. While the $x_{1}$ family is the backbone of thin or $2 \mathrm{D}$ bars, it is the whole $x_{1}$ tree, including the vertical families of periodic orbits bifurcating from the $x_{1}$ at various vertical resonances, that is the backbone of $3 \mathrm{D}$ bars and peanuts (Skokos, Patsis \& Athanassoula, 2002a). 


\subsection{The Role of the halo}

The halo had initially been thought to stabilise the disc and to postpone, if not stop, the growth of the bar (Athanassoula, 1984; Sellwood $\&$ Wilkinson, 1993; and references in either). Yet the results in Athanassoula \& Misiriotis (2002) and Athanassoula (2002a) show strong bars in simulations in which the disc is initially embedded in a very massive halo. Athanassoula (2002b) suggested that this is due to the response of the halo. Indeed, simulations with rigid haloes, whose mass within the disc region is a large fraction of the total mass within that volume, show that no strong bar is formed in a Hubble time; at the most a small oval in the central parts. On the other hand, if the halo is live it responds to the bar and takes angular momentum from it. This excites the bar, since the latter is a negative angular momentum 'perturbation', and makes it grow stronger. In particular, there is a considerable fraction of resonant particles/orbits in the halo component.

\subsection{Evolution of the STELlar COMPONENT}

Barred galaxies undergo considerable evolution even after the bar and peanut have formed. This is due to the fact that bars transfer angular momentum outwards (Lynden-Bell \& Kalnajs, 1972). Thus the disc/bar component loses angular momentum to the halo and the bar slows slows down (e.g. Weinberg, 1985; Little \& Carlberg, 1991a; Little \& Carlberg, 1991b; Hernquist \& Weinberg, 1992; Athanassoula, 1996; Debattista \& Sellwood, 1998; Debattista \& Sellwood, 2000; etc.). This slow-down is much more important if the halo contributes a considerable fraction of the mass within a sphere of radius equal to the disc radius (Debattista \& Sellwood, 1998). At the same time the bar becomes longer and stronger. A detailed, quantitative description of this evolution will be given elsewhere.

\subsection{Evolution due to the gaseous Component of the DisC}

Further types of evolution can be found when the galaxy has a sizeable gaseous component in its disc. Thus the spiral driven in the gas by the bar can evolve, due to the collisional nature of the gas. As shown initially by Schwarz (1981), this leads to the formation of rings at the main resonances, outer rings at the outer Lindblad resonance, inner ones at the iUHR and nuclear ones at the inner Lindblad resonance (hereafter ILR). Such rings can be long-lived.

The exchange of energy and angular momentum within the galaxy becomes more complicated than in cases with no gas, since the gas is

now a third partner in the exchange process. Thus it is possible for the 
bar to speed up somewhat, rather than slow down (e.g. Friedli \& Benz, 1993; Berentzen et al., 1998).

As a response to the bar forcing, the region in and around the bar is depleted of its gas, which concentrates in two narrow lanes along the leading edges of the bar (e.g. Athanassoula, 1992). These are in fact shock loci and, as a result the gas is driven inwards towards the central region. If the galaxy has no ILR, then the gas accumulates in a small region very near the center. This can also be the case if the galaxy has an ILR but the sound speed is very high, of the order of $30 \mathrm{~km} / \mathrm{sec}$ (Englmaier \& Gerhard, 1997; Patsis \& Athanassoula, 2000). If, on the other hand, the galaxy does have an ILR, as most barred galaxies probably do, and its sound speed is lower than $20 \mathrm{~km} / \mathrm{sec}$, as expected, then the gas occupies a large region of radius of the order of the ILR radius. How can the gas be brought further inwards to the innermost few parsecs, so that it can eventually fall on the nucleus and feed it and make it an active galactic nucleus? At least two ways have been so far proposed. Heller \& Shlosman (1994) propose that, when the central disc or ring becomes gas-dominated, it becomes gravitationally unstable and breaks into clumps, whose interactions and collisions may bring the gas further inwards towards the nucleus. A second alternative could be bars (or spirals) within bars, as initially proposed by Shlosman, Frank \& Begelman (1989). Recent hydrodynamical simulations, however, question this mechanism, arguing that secondary stellar bars are unlikely to increase the mass inflow rate into the galactic nucleus (Maciejewski et al., 2002). Further work on this subject is necessary to elucidate the properties and the role of the secondary bars.

When the gas reaches the center it will make a strong central concentration, whose effect may be to destroy the bar, or at least decrease its strength (Friedli \& Benz, 1993; Berentzen et al., 1998). Indeed Hasan \& Norman (1990) and Hasan, Pfenniger \& Norman (1993) have shown that a sufficiently strong central concentration will make the $x_{1}$ orbits unstable, so that a large fraction of the phase space will be occupied by chaotic orbits. Norman, Sellwood \& Hasan (1996) grew a massive core in the center of an $N$-body bar and showed that, provided this has a mass larger than $\sim 5 \%$ of the combined disc and bulge mass, it destroys the bar. However, the mass of black holes in disc galaxies (Gebhardt et al, 2000; Ferrarese \& Merritt, 2000) is smaller, by an order of magnitude or more, than the mass required by Norman, Sellwood \& Hasan, and thus may not be sufficient to destroy bars. 


\section{Interactions}

Let us now discuss a second engine which can drive evolution in disc galaxies, namely interactions. Contrary to bars, which drive a relatively slow evolution, interactions can drive a fast, and sometimes violent, evolution. There is a large variety of types of interactions and of possible results, of which we will review only a few specific cases.

\subsection{TAILS AND BRIDGES}

One of the most spectacular results of interactions is the formation of tails and bridges, such as observed e.g. in the Antennae (NGC 4038/4039), the Atoms-for-Peace (NGC 7252), or the Mice (NGC 4676). These structures are formed in a disc galaxy by the tidal influence of a companion galaxy, in particular in direct passages (Toomre \& Toomre, 1972). In such cases, the angular velocity of the companion is, temporarily, nearly equal to that of some of the stars in the disc of the target galaxy, and the effect of this 'broad resonance' can make the tidal tails particularly strong.

\subsection{Ring GALAXIES}

Another spectacular result of an interaction is the formation of rings. A spheroidal companion hitting a target disc galaxy near-perpendicularly and not far from its center will produce a density wave in form of an expanding ring (Lynds \& Toomre, 1976). Often a second ring appears after the first one and sometimes the two are linked by spokes (Appleton \& Struck-Marcell, 1996). A nice example of such a structure can be seen in the Cartwheel galaxy (A0035-324), which exhibits two rings and a number of spokes linking them. Companions of larger mass make primary rings of larger amplitude and width, which live longer and expand faster than rings made by less massive companions (Athanassoula, Puerari \& Bosma, 1997).

\subsection{INTERACTION OF A BARRED GALAXY AND A SMALL SPHEROIDAL COMPANION}

I will here summarise some results of a series of simulations of the interaction between a target barred disc galaxy and its spherical companion. The full analysis of these simulations has not yet been published, although preliminary results can be found in conference proceedings (Athanassoula 1996, 1999 and 2002a). Similar work, but for non-barred disc galaxies, has been published by Walker, Mihos \& Hernquist (1996), Huang \& Carlberg (1997) and Velazquez \& White (1999). 
Let us first consider the case where the companion is initially in a near-circular orbit on the equatorial plane of the target and its mass is equal to that of the disc of the target. It spirals fast towards the center of the disc, losing only a small fraction of its mass in the process. As it approaches the bar, it perturbs it strongly, so that a number of the particles orbiting in the bar are pulled towards the companion and the bar is progressively emptied and destroyed. During the interaction and subsequent merging the disc of the target thickens, but also expands, in such a way that it still remains a disc, albeit somewhat thickened. In the final stages of the evolution, after the companion has reached the center of the target, the bar is totally destroyed and the disc of the remnant is axisymmetric with a low density region in the center, which is occupied by the companion. Thus the companion either forms a bulge or contributes to a bulge, thus driving evolution of the target galaxy along the Hubble sequence from later towards earlier type disc galaxies.

Let us now consider a companion of mass equal to one tenth of the mass of the disc of the target and let its orbit again be near-circular and on the equatorial plane of the target. The evolution is totally different from that described previously for the case of a high mass satellite. The companion now takes considerably longer to spiral inwards and loses a large fraction of its mass in the process. Some of its particles stay in the outer parts of the disc forming a spiral structure; others get trapped in the outer parts of the bar around $x_{1}$-type orbits.

If the companion is initially on a near-circular orbit which forms an angle with the plane of the disc of the target, then the whole disc of the target tilts, but is not destroyed, even when the angle is of the order of $45^{\circ}$. For the massive companion case, the tilt angle is not far from the angle of the orbital plane of the companion, and it is considerably smaller for the case of the low mass companion.

\subsection{Mergings AND their Remnants}

When the encounter between two galaxies is sufficiently close and slow, then dynamical friction will bring them nearer and eventually lead to a merging (e.g. Toomre, 1977). Following the suggestion by Toomre \& Toomre (1972) that the merger remnant could be an elliptical, many studies have concentrated on comparing the properties of $N$-body merger remnants with those of observed elliptical galaxies (Barnes \& Hernquist, 1992; Barnes, 1998 and references therein). The results of such comparisons look encouraging, but many problems have still to be solved. Thus the radial projected density profiles display the $r^{1 / 4}$ form characteristic of elliptical galaxies over the main body of the rem- 
nant. In the center-most parts, however, these profiles do not have the correct form, unless the progenitor discs have little or no gas, as well as a considerable bulge component (Hernquist, 1992; Hernquist, 1993; Hernquist, Spergel \& Heyl, 1993; Mihos \& Hernquist, 1994). Also the angular momentum vector does not align with the minor axis of the remnant in cases of disc progenitors of equal mass. On the other hand, in cases of progenitors with 3:1 mass ratio the misalignment angles are much smaller, consistent with observations (Franx, Illingworth \& de Zeeuw, 1991). Whether these shortcomings are due to inadequacies of the present day modeling procedure, or whether they are pointing to inadequacies of the simple scenario where ellipticals would be due to merging of two equal mass galaxies having properties similar to those of disc galaxies at $z \sim 0$, is unclear.

Urged by such considerations, two independent studies turned next to multiple mergings and examined the structure of the resulting remnants. Weil \& Hernquist $(1994,1996)$ showed that the angular momentum vector of the remnant is well aligned with its minor axis, in good agreement with observations (Franx, Illingworth \& de Zeeuw, 1991), while Athanassoula \& Vozikis (1999) underlined a number of properties of the $N$-body remnant which are in agreement with those of elliptical galaxies. The two studies are complementary. The simulations of Weil \& Hernquist, far superior in particle number, have used only initial conditions preselected so that all their six galaxies would merge more or less simultaneously very early on in the simulation. This could influence the properties of the remnant, or, more precisely, could give information on only one type of merger remnant. On the other hand, Athanassoula \& Vozikis have a large variety of initial conditions, including both haloes common to the whole group and individual haloes around each galaxy, different halo-to-luminous mass ratios and different kinematics of the galaxies within the group (in virial equilibrium, collapsing, expanding or rotating). However, the number of particles they used was too low to allow them to calculate anything but global properties of the merger. Although they checked the robustness of their results by repeating some of their simulations with double the number of points, still a larger number of particles would be preferable.

For these reasons I started a series of simulations, as background jobs of our GRAPE-5 machines (Kawai et al., 2000), trying to couple the strong points of the two studies, i.e. the large particle number of Weil \& Hernquist with the larger variety of initial conditions of Athanassoula \& Vozikis. Preliminary results from this study show two distinct types of remnants. In most cases the merger remnant is a spheroidal object, whose properties are similar to those of the objects analysed by Weil \& Hernquist, i.e. resemble in many ways elliptical galaxies. There are, 
however, a few cases in which the disc nature of one of the progenitors is preserved, so that the remnant resembles more a Sombrero type S0 galaxy. Further analysis of this type of remnants, and of the initial conditions that lead to their formation, is underway.

\section{References}

Appleton, P. N. and C. Struck-Marcell. Collisional ring galaxies. Fund. Cosmic Phys., 16:111-220, 1996.

Athanassoula, E. The spiral structure of galaxies. Phys. Rep., 114:321-403, 1984.

Athanassoula, E. The existence and shape of dust lanes in galactic bars. MNRAS, 259:345-364, 1992.

Athanassoula, E. Evolution of bars in isolated and in interacting galaxies. In R. Buta, B. G. Elmegreen and D. A. Crocker, editors, Barred galaxies, pages 309-320. Publ. Astron. Soc. Pac. 91, 1996.

Athanassoula, E. The fate of barred galaxies in interacting and merging systems. In A. Sandqvist and P. O. Lindblad, editors, Barred galaxies and circumnuclear activity, pages 59-66, Lecture notes in physics 474, Springer-Verlag, 1996.

Athanassoula, E. $N$-body simulations of interacting disc galaxies. In J. A. Sellwood and J. Goodman, editors, Astrophysical discs, pages 351-359, Publ. Astron. Soc. Pac. 160, 1999.

Athanassoula, E. Isolated and interacting galaxies : simulations with GRAPE. In J. Makino and P. Hut, editors, Astrophysical supercomputing using particles, Astron. Soc. Pac. Conference Series, 2002a, in press.

Athanassoula, E. New results on bar-halo interactions. In G. S. Da Costa and E. M. Sadler, editors, The dynamics, structure and history of galaxies, Astron. Soc. Pac. Conference Series, 2002b, in press.

Athanassoula, E. and A. Misiriotis. Morphology, photometry and kinematics of $N$ body bars - I. Three models with different halo central concentrations. MNRAS, 330:35-52, 2002.

Athanassoula, E., I. Puerari and A. Bosma. Formation of rings in galactic discs by infalling small companions. MNRAS, 286:284-302, 1997.

Athanassoula, E. and Ch. L. Vozikis. The structure of merger remnants of compact groups of galaxies : some preliminary results. In J. E. Barnes and D. B. Sanders, editors, Galaxy interactions at low and high redshift, pages 145-148, Publ. Astron. Soc. Pac. 186, 1999.

Barnes, J. E. Dynamics of galaxy interactions. In D. Friedli, L. Martinet and D. Pfenniger, editors, Galaxies: interactions and induced star formation, pages 275-394, Springer-Verlag Berlin Heidelberg New York, 1998.

Barnes, J. E. and L. Hernquist. Dynamics of interacting galaxies. ARAEA, 30:705742, 1970.

Berentzen, I., C. H. Heller, I. Shlosman and K. J. Fricke. Gas-driven evolution of stellar orbits in barred galaxies. MNRAS, 300:49-63, 1998.

Binney, J. and S. Tremaine. Galactic dynamics. Princeton university press, Princeton, 1987.

Combes, F. and R. H. Sanders. Formation and properties of persisting stellar bars. AधA, 96:164-173, 1981.

Combes, F., F. Debbash, D. Friedli and D. Pfenniger. Box and peanut shapes generated by stellar bars. A\&A A, 233:82-95, 1990. 
Contopoulos G. How far do bars extend. A\& A, 81:198-209, 1980.

Contopoulos G. and P. Grosbøl. Orbits in barred galaxies. A\&AR, 30:705-742, 1989.

Debattista, V. P. and J. A. Sellwood. Dynamical friction and the distribution of dark matter in barred galaxies. ApJ, 493:L5-L8, 1998.

Debattista, V. P. and J. A. Sellwood. Constraints from dynamical friction on the dark matter content of barred galaxies. ApJ, 543:704-721, 2000.

Elmegreen, B. G. Pattern speeds in barred galaxies. In R. Buta, B. G. Elmegreen and D. A. Crocker, editors, Barred galaxies, pages 197-206. Publ. Astron. Soc. Pac. 91, 1996.

Englmaier, P. and O. Gerhard. Two modes of gas flow in a single barred galaxy. MNRAS, 287:57-68, 1997.

Ferrarese, L. and D. Merritt. A fundamental relation between supermassive black holes and their host galaxies. ApJ, 539:L9-L12, 2000.

Franx, M., G. Illingworth and T. de Zeeuw. The ordered nature of elliptical galaxies - Implications for their intrinsic angular momenta and shapes. ApJ, 383:112-134, 1991.

Friedli, D. and W. Benz. Secular evolution of isolated barred galaxies I. Gravitational coupling between stellar bars and interstellar medium. A\&SA, 268:65-85, 1993.

Gebhardt, K., R. Bender, G. Bower et al A relationship between nuclear black hole mass and galaxy velocity dispersion. ApJ, 539:L13-L16, 2000.

Hasan, H. \& Norman, C. Chaotic orbits in barred galaxies with central mass concentrations. ApJ, 361:69-77, 1990.

Hasan, H., D. Pfenniger \& C. Norman. Galactic bars with central mass concentrations - Three dimensional dynamics. ApJ, 409:91-109, 1993.

Heller, C. H. and I. Shlosman. Fueling nuclear activity in disc galaxies: Starbursts and monsters. ApJ, 424:84-105, 1994.

Hernquist, L. Structure of merger remnants I. Bulgeless progenitors. ApJ, 400:460475, 1992.

Hernquist, L. Structure of merger remnants II. Progenitors with rotating bulges. ApJ, 409:548-562, 1993.

Hernquist, L., D. N. Spergel and J. S. Heyl. Structure of merger remnants III. Phase-space constraints. ApJ, 416:415-424, 1992.

Hernquist, L. and M. L. Weil. Spokes in ring galaxies. MNRAS, 261:804-818, 1993.

Hernquist, L. and M. D. Weinberg. Bar-spheroid interactions in galaxies. ApJ, 400:80-95, 1992.

Huang, S. and R. G. Carlberg. Sinking satellites and tilting disc galaxies. ApJ, 480:503-523, 1997.

Kawai, A., T. Fukushige, J. Makino, and M. Taiji. GRAPE-5: A special purpose computer for $N$-body simulations. PASJ, 52:659-676, 2000.

Little, B. and R. G. Carlberg. The long-term evolution of barred galaxies. MNRAS, 250:161-170, 1991a.

Little, B. and R. G. Carlberg. Bar-disc angular momentum exchange. MNRAS, 251:227-242, 1991b.

Lynden-Bell, D. and A. J. Kalnajs. On the generating mechanism of spiral structure. MNRAS, 157:1-30, 1972.

Lynds, R. and A. Toomre. On the interpretation of ring galaxies : the binary ring system II Hz4. ApJ, 209:382-388, 1976.

Maciejewski, W., P. J. Teuben, L. S. Sparke \& J. M. Stone. Gas inflow in barred galaxies - Effects of secondary bars. MNRAS, in press, 2002 and astro-ph/0109431. 
Mihos, J. C. and L. Hernquist. Dense stellar cores in merger remnants. ApJ, 437:L47-L50, 1994.

Norman, C. A., J. A. Sellwood \& H. Hasan. Bar dissolution and bulge formation: an example of secular dynamical evolution in galaxies. ApJ, 462:114-124, 1996.

Patsis, P. and E. Athanassoula. SPH simulations of gas flow in barred galaxies. Effect of hydrodynamical and numerical parameters. A\&A, 358:45-56, 2000.

Raha, N., J. A. Sellwood, R. A. James and F. D. Kahn. A dynamical instability of bars in disk galaxies. Nature, 352:411-412, 1991.

Schwarz, M. P. The response of gas in a galactic disk to bar forcing. ApJ, 247:77-88, 1981.

Sellwood, J. A. and A. Wilkinson. Dynamics of barred galaxies. Rep. Prog. Phys., 56:173-255, 1993.

Shlosman, I., J. Frank and M. C. Begelman. Bars within bars - a mechanism for fueling active galactic nuclei. Nature, 338:45-47, 1989.

Skokos, H., P. Patsis and E. Athanassoula. Orbital dynamics of three-dimensional bars : I The backbone of 3D bars. A fiducial case. MNRAS, submitted, 2002a.

Skokos, H., P. Patsis and E. Athanassoula. Orbital dynamics of three-dimensional bars : II Investigation of the parameter space. MNRAS, submitted, 2002b.

Toomre, A. Mergers and some consequences. In B. M. Tinsley and R. B. larson, editors, The evolution of galaxies and stellar populations, pages 401-416, Yale University observatory, 1977.

Toomre, A. and J. Toomre. Galactic bridges and tails. ApJ, 178:623-666, 1972.

Velazquez, H. and S. D. M. White. Sinking satellites and the heating of galaxy discs. MNRAS, 304:254-270, 1999.

Walker, I. R., J. C. Mihos and L. Hernquist. Quantifying the fragility of galactic discs in minor mergers. ApJ, 460:121-135, 1996.

Weil, M. L. and L. Hernquist. Kinematic misalignments in remnants of multiple mergers. ApJ, 431:L79-L82, 1994.

Weil, M. L. and L. Hernquist. Global properties of multiple merger remnants. ApJ, 460:101-120, 1996.

Weinberg, M. D. Evolution of barred galaxies by dynamical friction. MNRAS, 213:451-471, 1985. 